\title{
A case of Pneumocystis jirovecii pneumonia in a patient with acquired immune defi- ciency syndrome who showed eosinophilia and an increased serum TARC/CCL17 level
}

\author{
Yuki Yabuuchi, ${ }^{1}$ Masashi Matsuyama, ${ }^{1}$ Sosuke Matsumura, ${ }^{1}$ Masayuki Nakajima, ${ }^{1}$ Yoshihiko Kiyasu, ${ }^{2}$ \\ Yuto Takeuchi, ${ }^{2}$ Yoshihiko Murata, ${ }^{3}$ Ryota Matsuoka, ${ }^{3}$ Masayuki Noguchi, ${ }^{3}$ Nobuyuki Hizawa ${ }^{1}$ \\ ${ }^{1}$ Department of Pulmonary Medicine; \\ ${ }^{2}$ Department of Infectious Diseases; \\ ${ }^{3}$ Department of Pathology, Faculty of Medicine, University of Tsukuba, Ibaraki, Japan
}

\begin{abstract}
Pneumocystis jirovecii pneumonia (PCP) in patients with acquired immune deficiency syndrome (AIDS) shows eosinophilic pneumonia like condition. The detailed mechanisms how AIDS-associated PCP causes eosinophilic pneumonia has not been elucidated, but it has been suggested that beta-D-glucan, a major component of Pneumocystis jirovecii, and Thelper type 2 immunity may be involved in the mechanism of eosinophilia in the lung. We experienced the case who developed an eosinophilic pneumonia-like condition in a patient with AIDS-associated PCP, whose clinical course indicated the importance of TARC/CCL17 but not IL-4 and IL- 5 as involved in eosinophilia caused by HIV and Pneumocystis jirovecii infection.
\end{abstract}

Key words: Pneumocystis jirovecii pneumonia; acquired immune deficiency syndrome; eosinophilia; eosinophilic pneumonia; TARC/CCL17.

Correspondence: Masashi Matsuyama, MD, PhD, 1-1-1 Tennoudai, Tsukuba, Ibaraki 305-8575, Japan.

Tel. +81.28.533144 - Fax: +81.28.537886. E-mail: mmatsuyama@md.tsukuba.ac.jp

Contributions: YY, drafted the manuscript; MM, designed experiments and analyses, conducted experiments, acquired and analyzed data, and edited the manuscript; YY, SM, MM, NH, examined the patient as pulmonologists; MN, conducted experiments; YT, YK, examined the patient as infectious control physicians; RM, YM, MN, performed pathological analysis; NH, revised the manuscript.

Conflict of interest: The authors declare that they have no competing interests, and all authors confirm accuracy.

Ethics approval: This study was approved by the Ethical Committee of the Faculty of Medicine, University of Tsukuba, Ibaraki, Japan.

Informed consent: Written informed consent was obtained from the patient for publication of this report and accompanying images. A copy of the written consent is available for review by the editor of this journal. 


\section{Introduction}

Human immunodeficiency virus (HIV) infection can cause eosinophilic inflammation [1,2]. In addition, Pneumocystis jirovecii pneumonia (PCP) in patients with acquired immune deficiency syndrome (AIDS) also shows an eosinophilic pneumonia (EP)-like condition [3-5]. Although the etiology of eosinophilia in AIDS has not been established, it has been argued that domains of HIV-1 proteins as antigens may induce T helper type 2 cells (Th2) via the signaling pathways containing cytokines, such as interleukin 4 (IL-4) and interleukin 5 (IL-5) [1]. For Pneumocystis jirovecii, an immune response to beta-D-glucan, a major component of its cell wall, may be involved in the mechanism of eosinophilia in the lung [6].

\section{Case presentation}

A case of PCP in an AIDS patient who showed eosinophilia in serum and bronchoalveolar lavage fluid (BALF) with increased levels of serum thymus and activation-regulated chemokine (TARC)/C-C motif chemokine ligand 17 (CCL17), but not of serum IL-4 and IL-5, is reported. The patient was a 36-year-old Chinese man with no remarkable past medical history. He had no history of smoking. He came to Japan two years ago as an agricultural trainee. He was admitted to our hospital with a complaint of increasing dyspnea. On presentation, the patient was fully conscious, with height $177 \mathrm{~cm}$, weight $58.9 \mathrm{~kg}$, temperature $35.8^{\circ} \mathrm{C}$, and $\mathrm{SpO}_{2} 89 \%$ on room air. On physical examination, decreased breath sounds were noted in the right lower lung. Laboratory data demonstrated eosinophilia (eosinophils: $1,067 / \mu \mathrm{l}$ ), mild lymphopenia (lymphocytes: 1,353/ $\mu \mathrm{l}$ ), elevated C-reactive protein (CRP: $1.93 \mathrm{mg} / \mathrm{dl}$ ), and a high serum level of Krebs von den Lungen-6 (KL-6: 1,277 U/ml). On high-resolution computed tomography (HRCT) of the chest, bilateral diffuse ground-grass opacities (GGOs), interlobular septal hypertrophy, and mild right pleural fluid were present (Figure 1A). There was no space to perform echo-guided thoracocentesis; therefore, an examination of pleural effusion was withdrawn. On examination of BALF obtained from the superior lingular bronchus, the total cell count was $1.70 \times 10^{5} / \mathrm{ml}$, comprised of $45.7 \%$ eosinophils, $36.8 \%$ lymphocytes, $2.6 \%$ neutrophils, and $14.9 \%$ macrophages. The increase in eosinophils in BALF suggested the presence of EP, whereas the decrease in the CD4/CD8 ratio (0.02) in BALF suggested the presence of HIV infection. Increased KL-6 was also a finding suggestive of HIV-positive PCP in the setting of increased eosinophils in BALF [3]. The serum anti-HIV-1 antibody test and HIV-1 Western blot analysis were positive. The amount of HIV-1 RNA in plasma was $6.2 \times 10^{4}$ copies $/ \mathrm{ml}$, indicating a high viral load. In addition,
A.

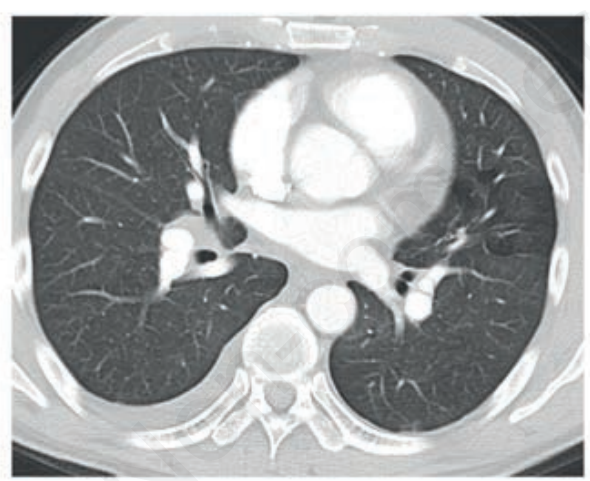

C.

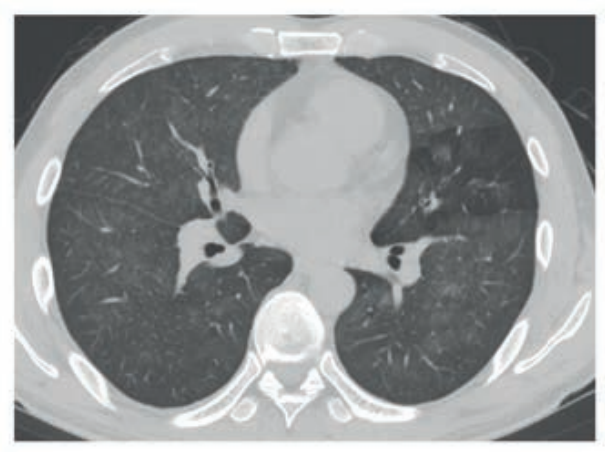

B.

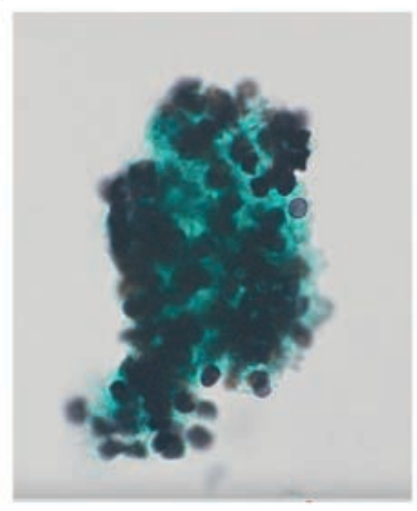

D.

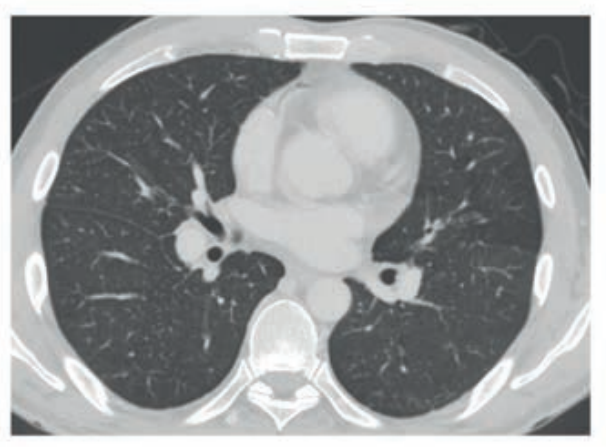

Figure 1. Imaging and pathological findings of this case. A) Bilateral ground-glass opacities and right pleural effusion on high-resolution computed tomography (HRCT) of the chest before starting treatment for PCP. B) Grocott stain $(\times 1000)$ of bronchoalveolar lavage fluid demonstrates uniformly sized and round yeast-like fungi. C) HRCT of the chest 15 days after PCP treatment shows that bilateral ground-glass opacities largely remain. D) HRCT of the chest 72 days after PCP treatment shows improvement of bilateral ground-glass opacities and the pleural effusion. 
there was a decrease in the peripheral blood CD4+ lymphocyte count $(68 / \mu \mathrm{l})$ and an increase in the $\beta$-D glucan level $(93.1 \mathrm{pg} / \mathrm{mL})$, and fungi stained with Grocott's method (Figure 1B) were noted in BALF. Based on these findings, the diagnosis was PCP and AIDS.

Serum levels of type 2 markers including IL-4, IL-5, TARC/CCL17, and total IgE were measured to establish the mechanism underlying the eosinophilic inflammation. A marked increase in serum TARC/CCL17 $[3,144 \mathrm{pg} / \mathrm{mL}$ (normal range $<450 \mathrm{pg} / \mathrm{ml}$ )] was found, whereas IL-4, IL-5, and total IgE were normal $[3.1 \mathrm{pg} / \mathrm{mL}(<6 \mathrm{pg} / \mathrm{ml}), 3.9 \mathrm{pg} / \mathrm{ml}(<3.9 \mathrm{pg} / \mathrm{ml})$ and 10 $\mathrm{IU} / \mathrm{m}(<170 \mathrm{IU} / \mathrm{ml})$, respectively]. To prevent the immune resuscitation inflammatory syndrome, the PCP was treated first. Sulfamethoxazole (SMX) 4,800 mg/trimethoprim (TMP) $960 \mathrm{mg}$ and oral corticosteroid $80 \mathrm{mg}$ were started, and the amount of corticosteroid was then gradually reduced. To prevent PCP recurrence, the patient was treated continuously with SMX 400 $\mathrm{mg} / \mathrm{TMP} 80 \mathrm{mg}$. After PCP treatment, bilateral GGOs and the right pleural effusion on chest HRCT improved gradually (Figure 1C, D). The number of eosinophils in blood decreased temporarily to $0 / \mu \mathrm{l}$ in response to steroids, and, at the same time, TARC/CCL17 in serum decreased to $1,970 \mathrm{pg} / \mathrm{ml}$. The time course of this case is shown in Figure 2. Twenty-eight days after the start of PCP treat- ment, fever and diarrhea appeared, and colonoscopy was performed, which showed intestinal tuberculosis in the ileocecal region. Pathological and bacteriological examinations confirmed intestinal tuberculosis. Treatment with rifampicin, ethambutol, isoniazid, and pyrazinamide was started, and the symptoms improved. Antiretroviral therapy (ART) consisting of emtricitabine, tenofovir, and raltegravir was then begun. Two weeks after the start of ART, the HIV-1 viral load decreased to $1.8 \times 10^{3}$ copies $/ \mathrm{mL}$, and the eosinophil count and TARC/CCL17 became normal, at $0 / \mu \mathrm{l}$, and $100 \mathrm{pg} / \mathrm{ml}$, respectively (Figure 2). He was discharged from our hospital and returned to China to continue his ART.

\section{Discussion}

Eosinophils in BALF can be increased in AIDS-related PCP [3-5]. The percentage of eosinophils in BALF in immunocompromised patients has been measured [5]; AIDS patients who developed PCP had a significant BALF eosinophilia compared to HIVinfected patients who did not develop PCP or non-HIV immunocompromised patients who developed PCP. HIV-1 envelopes may
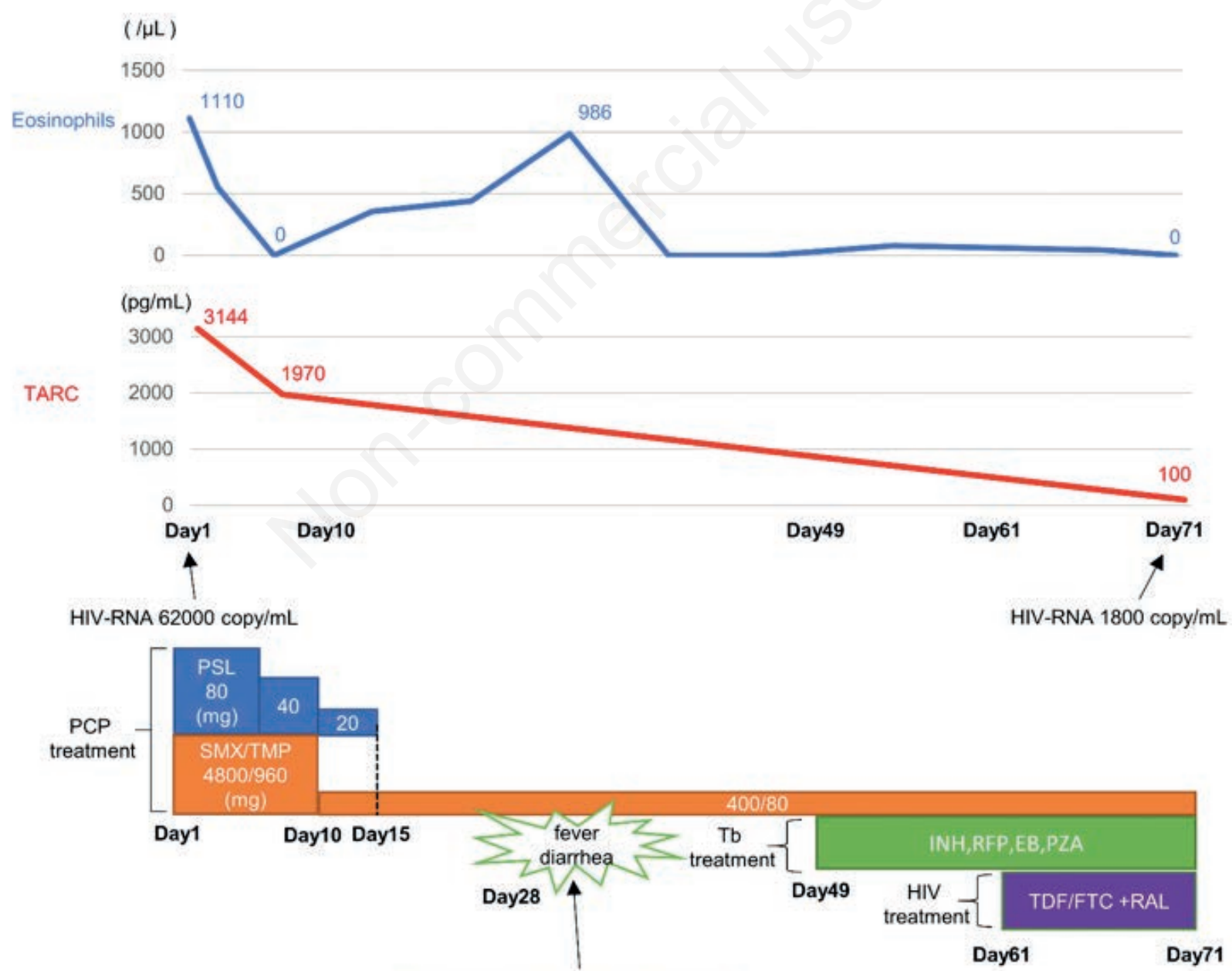

Intestinal tuberculosis was diagnosed

Figure 2. Time course of this case. INH, isoniazid; RFP, rifampicin; EB, ethambutol; PZA, pyrazinamide; TDF/FTC, tenofovir/emtricitabine; RAL, raltegravir; SMX/TMP, sulfamethoxazole/trimethoprim; PSL, prednisolone. 
induce the type- 2 immune response to stimulate IL-4 and IL-5 synthesis [1]. In the present case, the IL-4 and IL-5 levels were not elevated before PCP treatment, and both serum levels of TARC/CCL17 and blood eosinophil counts normalized along with the decrease in HIV copy number and improvement in the imaging findings of PCP, as shown in Figures 1 and 2. This clinical course suggested the importance of TARC/CCL17 rather than IL-4 and IL-5 in the eosinophilic inflammation in the present patient. Although peripheral blood eosinophils are rarely increased in tuberculosis, we cannot exclude the possibility that concomitant intestinal tuberculosis may affect TARC/CCL17 and peripheral blood eosinophilia, according to some reports $[7,8]$.

TARC/CCL17 is a chemokine associated with type 2 inflammation that induces eosinophilic inflammation [9]. It has been demonstrated that TARC/CCL17 was increased in HIV-1 infected patients [10]. TARC/CCL17 has been reported to be secreted from dendritic cells (DCs) stimulated by antigens, inducing Th2 cell activation [11]. One study also suggested that TARC/CCL17 itself, secreted from antigen-stimulated DCs, directly affects eosinophil recruitment without interaction with Th2 cells [12]. Because serum IL-4 and IL-5 levels were not elevated in the present case, the eosinophilic inflammation could have been induced directly by TARC/CCL17, which was secreted from DCs stimulated by infectious antigens, such as HIV-1 envelopes and beta-D-Glucan, from HIV and Pneumocystis jirovecii.

\section{Conclusion}

A case of an eosinophilic pneumonia-like condition in a patient with AIDS-associated PCP, whose clinical course showed the importance of TARC/CCL17, but not IL-4 and IL-5 in the eosinophilia caused by HIV and Pneumocystis jirovecii infection was presented.

\section{References}

1. Becker Y. The changes in the T helper 1 (Th1) and T helper 2 (Th2) cytokine balance during HIV-1 infection are indicative of an allergic response to viral proteins that may be reversed by Th2 cytokine inhibitors and immune response modifiers--a review and hypothesis. Virus Genes 2004;28:5-18.

2. Mayar M, Erick W, Roberto A, Thomas G, Jose S. Eosinophilia and associated factors in a large cohort of patients infected with human immunodeficiency virus. South Med J 2014; 107:554-8.

3. Ohji H, Shinohara T, Kadota N, Okano Y, Naruse K, Iwahara $\mathrm{Y}$, et al. Pneumocystis jirovecii pneumonia in an HIV-infected patient mimicking acute eosinophilic pneumonia: a case report with a review of the literature. J Thorac Dis 2018;10:E774-e8.

4. Allen JN, Davis WB, Pacht ER. Diagnostic significance of increased bronchoalveolar lavage fluid eosinophils. Am Rev Respir Dis 1990;142:642-7.

5. Fleury-Feith J, Van Nhieu JT, Picard C, Escudier E, Bernaudin JF. Bronchoalveolar lavage eosinophilia associated with Pneumocystis carinii pneumonitis in AIDS patients. Comparative study with non-AIDS patients. Chest 1989;95:1198-201.

6. Kawayama T, Fujiki R, Honda J, Rikimaru T, Aizawa H. High concentration of (1-->3)-beta-D-glucan in BAL fluid in patients with acute eosinophilic pneumonia. Chest 2003;123:1302.

7. Hansa H, Kibeten T, Teklu G, Hagos G. How common is eosinophilia in tuberculosis? Case Report. Pediatric Health Med Ther 2020;20:59-63.

8. Yonghong F, Hongyun Y, Guangliang M, Ling M, Heping X, Zhongyi H. Elevated serum levels of CCL17 correlate with increased peripheral blood platelet count in patients with active tuberculosis in China. Clin Vaccine Immunol 2011;18:629-32.

9. Miyazaki E, Nureki S, Ono E, Ando M, Matsuno O, Fukami T, et al. Circulating thymus- and activation-regulated chemokine/CCL17 is a useful biomarker for discriminating acute eosinophilic pneumonia from other causes of acute lung injury. Chest 2007;131:1726-34.

10. Malhotra P, Haslett P, Sherry B, Shepp DH, Barber P, Abshier $\mathrm{J}$, et al. Increased plasma levels of the TH2 chemokine CCL18 associated with low CD4+ T cell counts in HIV-1-infected patients with a suppressed viral load. Sci Rep 2019;9:5963.

11. Bonecchi R, Bianchi G, Bordignon PP, D'Ambrosio D, Lang $\mathrm{R}$, Borsatti A, et al. Differential expression of chemokine receptors and chemotactic responsiveness of type $1 \mathrm{~T}$ helper cells (Th1s) and Th2s. J Exp Med 1998;187:129-34.

12. Yi S, Zhai J, Niu R, Zhu G, Wang M, Liu J, et al. Eosinophil recruitment is dynamically regulated by interplay among lung dendritic cell subsets after allergen challenge. Nat Commun

\footnotetext{
Received for publication: 5 September 2021. Accepted for publication: 22 November 2021.

This work is licensed under a Creative Commons Attribution-NonCommercial 4.0 International License (CC BY-NC 4.0).

CC Copyright: the Author(s), 2022

Licensee PAGEPress, Italy

Multidisciplinary Respiratory Medicine 2022; 17:802

doi:10.4081/mrm.2022.802
} 